\title{
Acceptability of a Rinse Screening Test for Diagnosing Head and Neck Squamous Cell Carcinoma Among Black Americans
}

\author{
Suzanne C. Lechner • Lutécia Pereira • Erika Reategui • Claudia Gordon • \\ Margaret Byrne • Monica Webb Hooper • David J. Lee • Marianne Abouyared • \\ Elizabeth Franzmann
}

Received: 1 May 2014 /Revised: 9 July 2014 / Accepted: 22 August 2014 / Published online: 11 September 2014

(C) W. Montague Cobb-NMA Health Institute 2014

\begin{abstract}
Objective Head and neck squamous cell carcinoma (HNSCC) is a debilitating and deadly disease. We evaluated an easy-toadminister and innovative rinse that assays soluble CD44 and total protein as HNSCC early detection markers. We examined whether the rinse was acceptable and whether the results would promote screening behavior.

Study Design This is a prospective observational study. Methods Participants $(N=150)$ from underserved, lowincome Black American backgrounds completed assessments of satisfaction, intention to repeat test, and likely screening behavior after receiving results. Descriptive statistics, $t$ tests, and analysis of variance (ANOVA) were conducted.

Results The rinse was highly acceptable to participants and perceived to be acceptable among peers. Participants strongly agreed that they would perform the rinse as prescribed, engage in preventative behaviors if results indicated risk of cancer, and initiate treatment if they had a positive cancer finding.
\end{abstract}

\section{S. C. Lechner}

Department of Psychiatry and Behavioral Sciences, University of Miami Miller School of Medicine, Miami, FL, USA

S. C. Lechner $\cdot$ L. Pereira $\cdot$ E. Reategui $\cdot$ C. Gordon $\cdot$ M. Byrne $\cdot$ M. W. Hooper • D. J. Lee

Sylvester Comprehensive Cancer Center, Miami, FL, USA

M. Byrne • D. J. Lee

Department of Public Health Sciences, University of Miami Miller School of Medicine, Miami, FL, USA

M. W. Hooper

Department of Psychology, University of Miami, Miami, FL, USA

M. Abouyared $(\varangle) \cdot$ E. Franzmann

Department of Otolaryngology, University of Miami Miller School of Medicine, 1120 NW 14th Street 5th Floor, Miami, FL 33136, USA

e-mail: mabouyared@med.miami.edu
Employed participants slightly disliked the taste of the rinse but were more likely to schedule a follow-up appointment and engage in preventative behaviors based on the results. Those with health-care coverage (including public health insurance) reported that the test was harder to perform than those who were uninsured.

Conclusion An easy-to-use rinse technique is acceptable and likely to promote screening behavior among Black Americans at risk for HNSCC. Given that many cancer screening modalities are considered unpleasant to undergo, this rinse holds promise for promoting screening behaviors and, thereby, may result in early detection of this potentially fatal disease.

Level of Evidence: IV

Keywords Cancer screening $\cdot$ Head and neck cancer . Screening rinse $\cdot \mathrm{HNSCC} \cdot$ Black Americans

\section{Introduction}

Head and neck squamous cell carcinoma (HNSCC) is a debilitating and deadly disease. HNSCC includes cancers involving the oral cavity, pharynx, and larynx [1]. The Surveillance, Epidemiology, and End Results (SEER) database estimates a 5-year relative survival rate of $62.7 \%$ for oral cavity and pharynx cancers [2]. HNSCC tumors are often not discovered until a later stage, where the tumor is larger and/or has spread to other sites. Later stage disease thereby necessitates treatment that is likely to result in facial disfigurement and severe problems with speech, swallowing, and breathing $[3,4]$. Current avenues of research are thus focusing on molecular targets for early diagnosis and treatment to reduce the excess burden of disease on participants, their caregivers, and the US health-care system. 
HNSCC is particularly alarming among Black Americans, where there is a lower incidence of disease compared to other racial/ethnic groups, but higher mortality rates [5]. Reasons for the high mortality and morbidity of disease in this population subgroup include factors such as smoking and use of tobacco products, poverty-related factors, and eating a traditional cultural diet [6]. In South Florida, there is diversity even among those who self-identify as Black, because individuals who reside in this area may have roots in Caribbean nations (such as Bahamas, Jamaica, or Haiti) or be of AfricanAmerican cultural heritage. For this reason, we use the term "Black American" throughout this manuscript to pay respect to the fact that not all of our participants classified themselves as African-American. As such, we recognize that our participants had a heterogeneous set of cultural beliefs, expectations, and trust in the US health-care system [7].

Cure rates following diagnosis of HNSCC increase from 30 to $80 \%$ if the disease is detected early, emphasizing the importance of screening [8-10]. However, adoption of screening protocols can be hampered by the aversive aspects of the procedure. For example, fear of pain and discomfort often presents a barrier to screenings, such as mammography or digital rectal exams for breast cancer or prostate cancer screening, respectively [11-14]. Other routine cancer screening procedures (e.g., colonoscopies) are invasive, time-intensive, and perceived as burdensome, all of which may decrease screening behavior [15]. We sought to understand perceptions regarding the experimental rinse to detect HNSCC as preliminary evidence that this method of screening has the potential to be adopted in clinical practice settings.

Relatedly, there is a social phenomenon that accompanies the use of screening procedures. People often discuss their experiences during screening with friends and family, usually with the intention of promoting screening behavior in others [16]. However, if they highlight a negative aspect of the experience (i.e., the discomfort associated with colonoscopy prep), some individuals will respond by delaying, rather than initiating screening $[16,17]$. During the development of the screening rinse for HNSCC, we considered the aforementioned factors. We measured participants' experience with the screening protocol and also considered what people might report to their friends and family about the process to help us determine whether the newly developed tool would be acceptable on an individual and societal level as we move toward widespread dissemination.

Acceptability of screening procedures is of particular importance in low-income and racial minority population subgroups [16]. Screening rates among low-income Black Americans are suboptimal for procedures such as mammography and colorectal cancer screening [18, 19]. Underutilization of screenings result from perceived risk of cancer, fear of false positives, lack of overt symptoms, lack of knowledge, attitudes toward screening, beliefs about cancer, and financial burdens, among other factors [20]. Thus, in the development of a new screening protocol for cancer, it was important to consider attitudes toward screening, the perceived acceptability of the screening tool procedure, and whether the tool would be perceived as acceptable once the screening tool becomes a component of the standard of care. Information regarding the acceptability of the rinse protocol can inform future education-based interventions among Black Americans who are at very high risk for developing HNSCC.

This study evaluated the acceptability of a noninvasive, easy-to-administer, affordable, and innovative rinse technique that assays the soluble form of CD44 and total protein as HNSCC early detection markers in a sample of Black Americans. Previous research has shown that soluble CD44 and total protein levels are elevated in patients with HNSCC, and these levels may be associated with poorer outcomes [21, 22] The aim of the study was to determine the acceptability of the rinse and to understand whether the outcome of the rinse would be likely to promote screening behavior. In addition, we examined several a priori subgroup analyses to examine the acceptability of the rinse and the rinse's potential effects on behavior. We hypothesized that there would be no statistically significant group differences based on (a) gender, (b) educational attainment, (c) employment status, or (d) insurance status such that all groups would rate the rinse protocol as (a) highly acceptable and (b) likely to impact future behavior.

\section{Materials and Methods}

The study protocol was approved by the Institutional Review Board at the University of Miami. Study activities took place in the Liberty City neighborhood of Miami, at the Sylvester Comprehensive Cancer Center, the University of Miami Hospital and Clinics (UMHC), and Jackson Memorial Hospital (JMH) Otolaryngology Clinics. All participants provided written informed consent of all study procedures according to the Code of Ethics of the World Medical Association (Declaration of Helsinki).

\section{Study Participants}

The convenience sample included 150 Black American adults who resided in the Liberty City community in South Florida. Liberty City, which is considered an impoverished neighborhood, was specifically targeted for this study due to its potential excess burden of HNSCC, our collaborative community partnership with the community, and its proximity to our medical center. Potential participants were recruited from a community center and a food bank and were scheduled for an appointment to meet with a research coordinator to perform the rinse and questionnaires. Potential participants were required to be high risk for HNSCC (defined as over 40 years of 
age and who identified themselves as active users of tobacco and/or alcohol). Patients with active cancers (except nonmelanoma skin cancer) were excluded from the study.

A study recruiter was identified, trained, and supervised by the Disparities and Community Outreach Shared Resource of the University of Miami Sylvester Comprehensive Cancer Center. She received extensive training in the protection of human subjects and how to obtain informed consent without coercion. All study recruitment activities were monitored by both the IRB and the Disparities and Community Outreach Shared Resource.

\section{Survey}

Study participants were administered a questionnaire to assess satisfaction with the rinse, intention to repeat the test, and whether the results would affect screening behavior. Content of the questionnaire is presented in Table 1 as a mean value with standard deviation in parenthesis. Participants were instructed to rate each items on a four-point scale (1 being strongly agree to 4 being strongly disagree). Mean value was calculated based on all participants' responses. The item set for the questionnaire was created specifically for the purposes of this study using classical test theory techniques. The questionnaire has not been subjected to psychometric testing; however, it was designed to have maximum face validity. The questionnaire assessed two domains: (a) acceptability of rinse protocol for self and social group and (b) the potential effects on screening behavior. Acceptability items included the following: agreement with the procedure of using a rinse to detect HNSCC, perceived difficulty of the rinse protocol, opinions of the taste of the rinse solution, willingness to perform the rinse protocol again, perception that others in his/her social group would find the rinse protocol easy to complete, and perceived willingness of peers to perform the rinse protocol. The potential effects on screening behavior included whether participants would schedule an appointment for treatment if the results were indicative of cancer, follow screening recommendations, take preventative action to prevent cancer based on screening results, and stop smoking to prevent cancer based on screening results. One item measured whether people at risk for HNSCC should stop smoking. Descriptive statistics, $t$ tests, and analysis of variance (ANOVA) were conducted using SPSS 21 (IBM).

In addition to the rinse-specific questionnaire, study subjects were administered a self-report questionnaire to collect data regarding their demographics (age, gender, race, educational attainment, and insurance coverage), health status, oral health, medical history, family history of HNSCC, nutrition, tobacco exposure (personal use or passive smoke exposure), and alcohol use. Portions of our tobacco exposure questions were derived from a questionnaire from the Center for Tobacco Control Research and Education. Age, education, employment, and insurance status were used as a priori stratification variables in the statistical analyses that follow. The remainder of the variables was collected in order to characterize the sample and to provide context for the findings.

\section{Oral Rinse Test}

Oral rinses were collected using previously published procedures $[21,22]$. Participants refrained from smoking, drinking eating, and brushing teeth for $1 \mathrm{~h}$ prior to collection. Using $5 \mathrm{ml}$ normal saline, participants were instructed to rinse for $5 \mathrm{~s}$,

Table 1 A summary of study subjects' responses to questionnaire

\begin{tabular}{|c|c|c|c|c|}
\hline & Strongly agree & Agree & Disagree & Strongly disagree \\
\hline $\begin{array}{l}\text { It would be great if doctors could detect head and neck cancer using a swish } \\
\text { and gargle rinse }\end{array}$ & $1.27(0.63)$ & & & \\
\hline The rinse was hard to do & & & $3.42(0.74)$ & \\
\hline The rinse tasted bad & & & $2.83(0.94)$ & \\
\hline I would not want to do the rinse again & & & $3.25(0.77)$ & \\
\hline It would be easy for other people I know to use the rinse & & $1.73(0.85)$ & & \\
\hline $\begin{array}{l}\text { Other people I know wouldn't mind using the rinse to detect cancer in the } \\
\text { head and neck }\end{array}$ & & $1.59(0.81)$ & & \\
\hline $\begin{array}{l}\text { If the results of the rinse said I might develop cancer, I would schedule an } \\
\text { appointment for treatment }\end{array}$ & $1.33(0.69)$ & & & \\
\hline $\begin{array}{l}\text { If I knew the rinse could detect cancer in my head or neck, I would do the } \\
\text { rinse as often as my doctor prescribed }\end{array}$ & $1.25(0.57)$ & & & \\
\hline $\begin{array}{l}\text { If the rinse said I had a high chance of getting head and neck cancer, I would } \\
\text { take action to prevent getting cancer }\end{array}$ & $1.33(0.66)$ & & & \\
\hline $\begin{array}{l}\text { If the rinse said I had a high chance of getting head and neck cancer, and I knew } \\
\text { that stopping smoking could prevent me from getting cancer, I would stop smoking }\end{array}$ & $1.27(0.59)$ & & & \\
\hline People who are at risk of head and neck cancer should stop smoking & $1.27(0.61)$ & & & \\
\hline
\end{tabular}

$N=150$. Text represents mean scores with standard deviations in parentheses 
gargle for $5 \mathrm{~s}$, and expectorate into a collection tube. The oral rinse test and questionnaires were completed during the same appointment.

\section{Results}

Participants ( $N=150 ; 88$ males and 62 females) were Black American men and women from underserved, low-income backgrounds. Eighty-seven percent of the sample reported an annual income of less than $\$ 20,000$, and the remaining $13 \%$ chose not to report their income. Participants were predominantly middle aged (mean age $=51.2, \mathrm{SD}=5.73$, range 40-70). One participant considered himself to be from a Black Hispanic background. Nearly all participants (98.7\%) were currently smoking or using nicotine products. As shown in Table 1, using the rinse was highly acceptable to Black American men and women. With regard to the perceived acceptability of the rinse protocol for oneself and social group, participants strongly agreed with the likeability of a procedure that used a rinse to detect HNSCC. Overall, participants did not perceive difficulty using the rinse protocol, they rated the taste of the rinse solution as mildly negative, and they were willing to perform the rinse protocol again. There was general agreement that others in their social group would find the rinse to be easy to administer and would be willing to perform the tasks in the HNSCC screening protocol.

The rinse's potential effects on screening behavior were each rated in the "strongly agree" category. That is, participants strongly agreed that if the rinse results were positive for cancer, he/she would schedule an appointment for treatment, follow screening recommendations, take preventative action to prevent cancer based on screening results, and stop smoking to prevent cancer. Participants strongly agreed that people at risk for HNSCC should stop smoking.

We then conducted a priori subgroup analyses to examine the acceptability of the rinse and the rinse's potential effects on behavior among (a) men versus women, (b) by stratifications based on educational attainment, (c) employment status, and (d) insurance status.

\section{Gender Subgroup Analyses}

Men and women did not differ on any of the items reflecting acceptability of the rinse (all $p$ values of $t$ tests $>0.05$ ) or future behavior (all $p$ values of $t$ tests $>0.05$ ).

\section{Educational Attainment Categories}

Acceptability and future behavior indices did not differ based on participants' educational attainment (all $p$ values of ANOVAs $>0.05)$.
Employment

Compared to currently unemployed participants $(N=125)$, those who were employed $(N=25)$ reported displeasure with the taste of the rinse comprised of normal saline (employed mean score $=1.03$ versus unemployed mean score 0.89 ; $t(1,148)=-3.09, p<0.01)$. Employed participants would be more likely to schedule a treatment appointment based on the rinse's outcome (employed mean score $=1.64$ versus unemployed mean score $1.27 ; t(1,148)=2.47, p<0.05$. Employed participants also felt that they would be more likely to act to prevent HNSCC compared to unemployed participants (employed mean score $=1.60$ versus unemployed mean score $1.27 ; t(1,148)=2.30, p<0.05$. All other analyses were not statistically significant at the criterion of $p<0.05$.

\section{Insurance}

Individuals with health-care coverage reported that the test was harder to complete (insured mean score $=3.55$ out of a possible 4 versus uninsured mean score $=3.26 ; t(1,148)=2.39$, $p<0.05$ ). The groups did not have any other variables (all $p$ values of $t$ tests $>0.05$ ).

\section{Discussion}

In general, the US adult population is enthusiastic about cancer screening, with most individuals willing to undergo screening examinations even in cases where the illness is untreatable [23]. However, many constraints inhibit patients from seeking even the most established screening tests, such as those for colorectal cancer and breast cancer. Common deterrents include poor knowledge of disease importance, misinformation regarding the need for regular screening, cost of screening, and lack of insurance [24, 25]. Thus, there is great need for screening modalities that are low in cost and perceived as acceptable to individuals in the community. In this study, we examined participants' views of an easy-toadminister rinse technique to screen to HNSCC. We did not expect to see differences based on gender, educational attainment, employment status, or insurance status.

We were particularly interested in the effects of insurance status on the results of this study. According to the SEER data, Black American individuals have a higher mortality rate for HNSCC as compared to any other race. However, one study, which included patients solely treated at a Veterans Administration (VA) Hospital, examined laryngeal carcinoma survival and found no difference between Black American and Caucasian patients [26]. This may be attributable to the greater access and follow-up care that individuals have in the VA system as compared to other forms of public and private 
health care. The Roach et al. [26] study shows that the goal of attenuating health disparities faced by minority individuals is achievable and targets insurance and access to care as potential intervention points for modifying racially based health disparities. With this in mind, we examined whether insurance status affected perceptions of the rinse protocol and participants' intention for future behavior.

Based on our survey results, the rinse screening test, as reported in Franzmann [22], is easy to use and of low cost and can be easily accessible to patients and their providers. Our study found that a simple rinse technique was acceptable and likely to promote screening behavior among Black Americans at risk for HNSCC. Participants expressed positive attitudes toward the rinse, indicating its ease of use.

Compared to participants who were unemployed, employed participants more often indicated that they would follow up based on the rinse's outcome by seeking cancer treatment. This finding is consistent with Beeker et al. [24] and Hsia et al. [25] who cited concerns about cost and lack of adequate health insurance as possible deterrents for seeking cancer screening. It is likely that the group of employed participants in this study differed from the general population of employed Black Americans, given their low-income levels and their availability to participate in the study during the typical workday. We suspect that these individuals were perhaps working but underemployed, but this remains to be speculative. Furthermore, individuals with health-care coverage were more likely to indicate that the rinse was "hard to do". Because our definition of "insured" included patients with Medicare, Medicaid, prepaid plans, HMOs, and the Ryan White program (a state-run program for HIV+ individuals), using this variable as an indicator of access and healthcare coverage is not warranted. There are limitations in the generalizability of our findings, comparing insured versus uninsured and employed versus unemployed participants.

Our sample included people living in a community that largely consists of low-income, Black American individuals. Despite the statistic that Black Americans are less likely to smoke than non-Hispanic Whites [27], nearly $100 \%$ of our participants reported current smoking and low motivation to quit smoking. Given the rich data that smoking contributes to negative health outcomes and enhances the risk for HNSCC, there is great potential for a HNSCC screening test to positively impact this community. In this study, the majority of participants strongly agreed that if the rinse indicated a high risk of HNSCC, they would stop smoking. Thus, the perception of elevated risk, based on a simple rinse screening test could encourage adaptive health behavior in an underserved community.

In summary, this study yielded promising information about the acceptability of a simple, cost-effective screening modality for HNSCC. Participants indicated that the technique was easy to perform, that they were likely to perform routine screenings, and that the result would guide future behavior. Given that many cancer screening modalities are considered unpleasant to undergo (e.g., colonoscopies, mammograms), this new rinse screening tool holds promise for promoting screening behaviors, thereby resulting in early detection of this potentially fatal disease.

Acknowledgments Funding was provided by the State of Florida Bankhead Coley Cancer Research Program. We acknowledge the support of the University of Miami Sylvester Comprehensive Cancer Center, the University of Miami Miller School of Medicine Department of Otolaryngology, the Disparities and Community Outreach Shared Resource of the UM Sylvester Comprehensive Cancer Center, and the NonTherapeutic Research Support Shared Resource of the UM Sylvester Comprehensive Cancer Center. We thank our participants for their time and effort.

Financial Disclosure Information contained is subject to protection by issued patent and pending patent applications owned by the University of Miami. University of Miami and Dr. Franzmann may have the potential to benefit from future commercialization. Additionally, the government retains certain rights in these inventions.

Conflict of Interest Authors Suzanne C. Lechner, Lutécia Pereira, Erika Reategui, Claudia Gordon, Margaret Byrne, Monica Webb Hooper, David J. Lee, and Marianne Abouyared declare that they have no conflict of interest. Elizabeth Franzmann is an investor of the Intellectual Property licensed to Vigilant. She is also a consultant for Vigilant, holds equity in the company, and serves as the CSO.

Informed Consent All procedures followed were in accordance with the ethical standards of the responsible committee on human experimentation (institutional and national) and with the Helsinki Declaration of 1975 , as revised in 2000 (5). Written informed consent was obtained from all participants in the study.

\section{References}

1. Muir C, Weiland L. Upper aerodigestive tract cancers. Cancer. 1995;75(1 Suppl):147-53.

2. SEER cancer statistics factsheets: oral cavity and pharynx cancer. National Cancer Institute. Bethesda. http://seer.cancer.gov/statfacts/ $\mathrm{html} /$ oralcav.html

3. Al-Sarraf M. Treatment of locally advanced head and neck cancer: historical and critical review. Cancer Control. 2002;9(5):387-99.

4. Forastiere A, Koch W, Trotti A, Sidransky D. Head and neck cancer. N Engl J Med. 2001;345(26):1890-900.

5. DeSantis C, Naishadham D, Jemal A. Cancer statistics for African Americans, 2013. CA Cancer J Clin. 2013;63(3):151-66.

6. Goodwin WJ, Thomas GR, Parker DF, et al. Unequal burden of head and neck cancer in the United States. Head Neck. 2008;30(3):358 71.

7. Meyerowitz BE, Richardson J, Hudson SL, B. L. Ethnicity and cancer outcomes: behavioral and psychosocial considerations. Psychol Bull. 1998;123(1):47-70.

8. Sankaranarayanan R, Ramadas K, Thomas G, et al. Effect of screening on oral cancer mortality in Kerala, India: a cluster-randomised controlled trial. Lancet. 2005;365(9475):1927-33.

9. Sankaranarayanan R, Mathew B, Jacob BJ, et al. Early findings from a community-based, cluster-randomized, controlled oral cancer 
screening trial in Kerala, India. The Trivandrum Oral Cancer Screening Study Group. Cancer. 2000;88(3):664-73.

10. Brocklehurst P, Kujan O, Glenny AM, et al. Screening programmes for the early detection and prevention of oral cancer. Cochrane Database Syst Rev. 2010;(11):CD004150.

11. Aro AR, de Koning HJ, Absetz P, Schreck M. Two distinct groups of non-attenders in an organized mammography screening program. Breast Cancer Res Treat. 2001;70(2):145-53.

12. Consedine NS, Magai C, Krivoshekova YS, Ryzewicz L, Neugut AI. Fear, anxiety, worry, and breast cancer screening behavior: a critical review. Cancer Epidemiol Biomarkers Prev. 2004;13(4):501-10.

13. Nijs HG, Essink-Bot ML, DeKoning HJ, Kirkels WJ, Schroder FH. Why do men refuse or attend population-based screening for prostate cancer? J Public Health Med. 2000;22(3):312-6.

14. Myers RE, Wolf TA, McKee L, et al. Factors associated with intention to undergo annual prostate cancer screening among African American men in Philadelphia. Cancer. 1996;78(3): 471-9.

15. de Wijkerslooth TR, de Haan MC, Stoop EM, et al. Burden of colonoscopy compared to non-cathartic CT-colonography in a colorectal cancer screening programme: randomised controlled trial. Gut. 2012;61(11):1552-9.

16. Ogedegbe G, Cassells AN, Robinson CM, et al. Perceptions of barriers and facilitators of cancer early detection among lowincome minority women in community health centers. J Natl Med Assoc. 2005;97(2):162-70.

17. Peek ME, Sayad JV, Markwardt R. Fear, fatalism and breast cancer screening in low-income African-American women: the role of clinicians and the health care system. J Gen Intern Med. 2008;23(11): 1847-53.
18. Campbell MK, James A, Hudson MA, et al. Improving multiple behaviors for colorectal cancer prevention among African American church members. Health Psychol. 2004;23(5):492-502.

19. Crump SR, Mayberry RM, Taylor BD, Barefield KP, Thomas PE. Factors related to noncompliance with screening mammogram appointments among low-income African-American women. J Natl Med Assoc. 2000;92(5):237-46.

20. Young RF, Severson RK. Breast cancer screening barriers and mammography completion in older minority women. Breast Cancer Res Treat. 2005;89(2):111-8.

21. Franzmann EJ, Reategui EP, Pereira LH, et al. Salivary protein and solCD44 levels as a potential screening tool for early detection of head and neck squamous cell carcinoma. Head Neck. 2012;34(5):687-95.

22. Franzmann EJ, Reategui EP, Pedroso F, et al. Soluble CD44 is a potential marker for the early detection of head and neck cancer. Cancer Epidemiol Biomarkers Prev. 2007;16(7):1348-55.

23. Schwartz LM, Woloshin S, Fowler Jr FJ, Welch HG. Enthusiasm for cancer screening in the United States. JAMA. 2004;291(1):71-8.

24. Beeker C, Kraft JM, Southwell BG, Jorgensen CM. Colorectal cancer screening in older men and women: qualitative research findings and implications for intervention. J Community Health. 2000;25(3):263-78.

25. Hsia J, Kemper E, Kiefe C, et al. The importance of health insurance as a determinant of cancer screening: evidence from the women's health initiative. Prev Med. 2000;31(3):261-70.

26. Roach 3rd M, Alexander M, Coleman JL. The prognostic significance of race and survival from laryngeal carcinoma. J Natl Med Assoc. 1992;84(8):668-74.

27. Trinidad DR, Perez-Stable EJ, White MM, Emery SL, Messer K. A nationwide analysis of US racial/ethnic disparities in smoking behaviors, smoking cessation, and cessation-related factors. Am J Public Health. 2011;101(4):699-706. 